\title{
Continuous-wave infrared optical gain and amplified spontaneous emission at ultralow threshold by colloidal HgTe quantum dots
}

\author{
Pieter Geiregat ${ }^{1,2,3 \star}$, Arjan J. Houtepen ${ }^{1,4}$, Laxmi Kishore Sagar ${ }^{1,3}$, Ivan Infante ${ }^{5}$, Felipe Zapata ${ }^{5}$, \\ Valeriia Grigel ${ }^{1,3}$, Guy Allan ${ }^{6}$, Christophe Delerue ${ }^{6}$, Dries Van Thourhout ${ }^{2,3}$ and Zeger Hens ${ }^{1,3}$
}

\begin{abstract}
Colloidal quantum dots (QDs) raise more and more interest as solution-processable and tunable optical gain materials. However, especially for infrared active QDs, optical gain remains inefficient. Since stimulated emission involves multifold degenerate band-edge states, population inversion can be attained only at high pump power and must compete with efficient multi-exciton recombination. Here, we show that mercury telluride (HgTe) QDs exhibit size-tunable stimulated emission throughout the near-infrared telecom window at thresholds unmatched by any QD studied before. We attribute this unique behaviour to surface-localized states in the bandgap that turn HgTe QDs into 4-level systems. The resulting longlived population inversion induces amplified spontaneous emission under continuous-wave optical pumping at power levels compatible with solar irradiation and direct current electrical pumping. These results introduce an alternative approach for low-threshold QD-based gain media based on intentional trap states that paves the way for solution-processed infrared QD lasers and amplifiers.
\end{abstract}

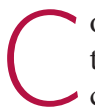
olloidal quantum dots (QDs) combine size-tuneable electronic energy levels with a suitability for solution-based processing, priming them for numerous optoelectronic applications $^{1,2}$. QD photodetectors ${ }^{3}$, spectrometers ${ }^{4}$, solar cells ${ }^{5}$, white light-emitting diodes (LEDs) and displays ${ }^{6}$ rely on the broad QD absorption spectrum with its size-dependent onset corresponding to the lowest energy exciton (X) transition and/or the tunable, narrow emission spectrum resulting from exciton recombination. Application development is further driven by unique characteristics such as multi-X generation ${ }^{7}$ or room-temperature single-photon emission $^{8}$ and the possibility to fine-tune optoelectronic properties by heterostructure formation ${ }^{9}$, doping ${ }^{10}$ or alloying ${ }^{11}$.

Tunable, low-cost and solution-processable gain media can equally impact on devices such as lasers or amplifiers ${ }^{12}$. Telecom applications operating at wavelengths around 1.3 or $1.55 \mu \mathrm{m}$, for example, make use of expensive epitaxially grown III-V lasers. Integrated photonics platforms based on silicon or silicon nitride, whereby small footprint photonic integrated circuits for datacommunication, signal processing and sensing are fabricated using CMOS-compatible processes ${ }^{13,14}$ even lack a native gain medium. In the case of silicon photonics, solutions relying on III-V die bonding, direct III-V epitaxy on silicon ${ }^{15,16}$, erbium doping ${ }^{17,18}$ or strained $\mathrm{Ge}^{19}$ are heavily investigated but suffer from issues with cost, upscaling, efficiency or limited gain bandwidth. A solutionprocessable gain medium based on colloidal QDs can solve such issues, as recently demonstrated for silicon nitride photonics ${ }^{20}$.

Stimulated emission involving the bandgap transition of QDs has been amply demonstrated ${ }^{21-24}$. Their use as gain media, however, remains limited since stimulated emission and absorption involve transitions between the same discrete band-edge states ${ }^{12,25}$. Since these are degenerate, QDs must contain more than one exciton (X) on average to achieve population inversion ${ }^{21}$. Next to this intrinsic drawback of two-level gain media, multi-X rapidly recombine through non-radiative Auger processes ${ }^{26}$. The population-inversion lifetime is therefore limited to a few tens of picoseconds, which means that gain thresholds are often reached only by femtosecond pulsed lasers ${ }^{27}$. Strategies to circumvent the limitations of multi-X gain involve slowing down Auger recombination through interfacial alloying in core/shell QDs and/or using large volume QDs, ${ }^{28}$ strain $^{29}$ or two-dimensional platelets ${ }^{25}$. Alternatively, single-X gain has been demonstrated using so-called type II core/shell QDs ${ }^{22}$, where repulsive $\mathrm{X}-\mathrm{X}$ interactions lift the effective level degeneracy. Unfortunately, both approaches are restricted to a few materialstypically involving Cd-based QDs emitting in the visible-and gain thresholds remain incompatible with, for example, direct-current electrical pumping ${ }^{25}$.

Here, we report on optical gain in dispersions of mercury telluride ( $\mathrm{HgTe}$ ) QDs and amplified spontaneous emission in $\mathrm{HgTe}$ QD polymer composites with average excitation densities $\langle N\rangle$ as low as 0.005 per QD, meaning that optical gain is attained by exciting only 1 out of 200 QDs in an ensemble. Intrinsic gain coefficients of $100-500 \mathrm{~cm}^{-1}$ are demonstrated and no decay of the population inversion is seen within the first nanoseconds after photoexcitation. We attribute this almost thresholdless gain to stimulated emission between the lowest conduction-band level and a shallow, empty state in the bandgap. The ensuing 4-level system results in high gain thresholds under continuous-wave (CW) optical pumping that now meet the requirements for direct current (DC) electrical pumping ${ }^{30}$. 


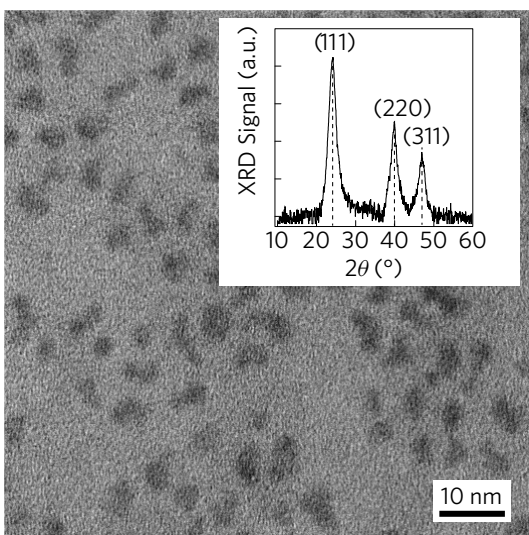

C

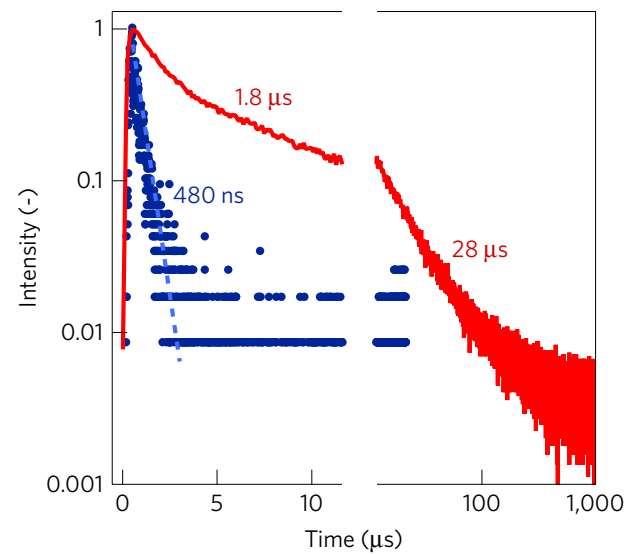

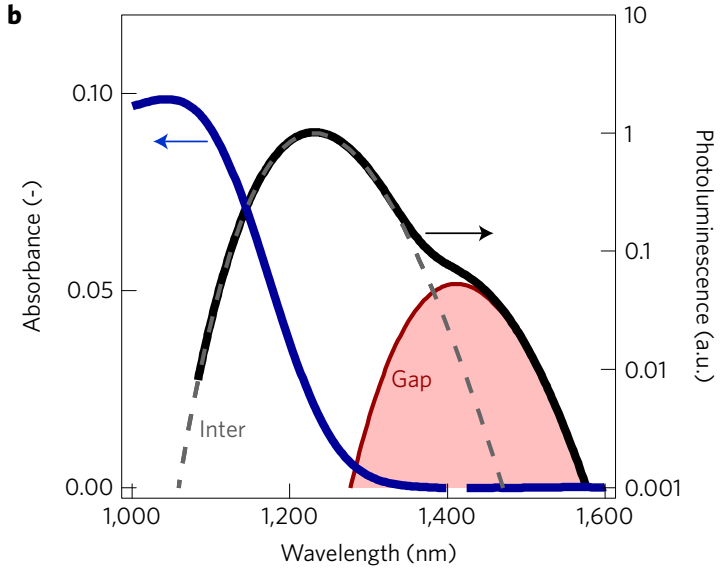

d

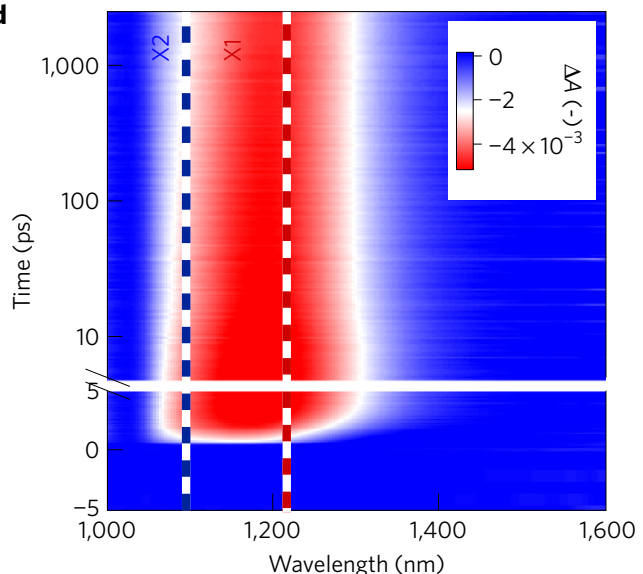

Figure 1 | Structural and linear optical properties of HgTe quantum dots (QDs). a, Transmission electron microscopy (TEM) image of $3.5 \pm 0.3$ nm

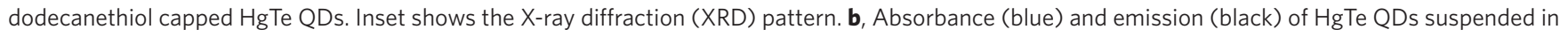
TCE as used in the transient absorption experiments. A clear additional emission band at lower energy is resolved (red) strongly red-shifted from the

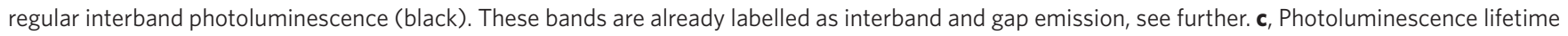
after excitation with $9 \mathrm{~ns}$ pulses at $532 \mathrm{~nm}$ for emission at the band edge (1.1 eV, blue) or in the low-energy band ( $0.85 \mathrm{eV}$, red). The average lifetime (see Methods) is determined to be 480 ns for the band-edge component and 1.8 to $28 \mu$ s for the emission band at lower energy. $\mathbf{d}$, Two-dimensional transient absorption $(\Delta A)$ map as a function of wavelength and time, showing the broad bleach feature $(\Delta A<0)$ obtained after 700 nm photoexcitation at $t=0$ with average number of absorbed photons per nanocrystal $\langle N\rangle=0.1$. The band-edge features $X_{1}$ and $X_{2}$ as predicted by tight-binding calculations (see Supplementary Section 2) are added for comparison.

\section{Basic properties of $\mathrm{HgTe}$ quantum dots}

$\mathrm{HgTe}$ is a semi-metal with a direct negative bulk bandgap. Size quantization enables the bandgap of $\mathrm{HgTe}$ QDs to be tuned from the long-wave infrared down to the near-infrared, reaching approximately $1 \mathrm{eV}$ for 3-4 $\mathrm{nm}$ QDs $^{31}$. The colloidal HgTe QDs used here have been synthesized according to a modified literature procedure $^{32,33}$ (see Methods) leading to QDs with the zinc-blende crystal structure (see Fig. 1a). Their absorption spectrum exhibits a clear first exciton transition with a size-tunable maximum attained at approximately $1,100 \mathrm{~nm}$ for the example shown in Fig. 1b. The QD photoluminescence spectrum, on the other hand, is composed of a high intensity band peaking at approximately $1,225 \mathrm{~nm}$, which we attribute to band-edge recombination and a low-intensity, subbandgap feature red-shifted by $100-150 \mathrm{meV}$ relative to the bandedge emission. This combination of two emission bands is more pronounced at low temperature (see Supplementary Section 1). Importantly, while the band-edge emission has a luminescent lifetime of approximately $400 \mathrm{~ns}$, the sub-bandgap transition shows a strongly delayed, multi-exponential emission that only drops to $1 \%$ of the original intensity after $100 \mu$ s (see Fig. 1c).

According to tight-binding calculations (see Supplementary Section 2) ${ }^{34,35}$, the band-edge absorption of HgTe QDs consists of two exciton transitions, labelled $\mathrm{X}_{1}$ and $\mathrm{X}_{2}$, between the lowest conduction-band level $\left(\mathrm{CB}_{1}\right)$ and the two uppermost valence-band levels $\left(\mathrm{VB}_{1}, \mathrm{VB}_{2}\right)$. Photoexcitation with a $700 \mathrm{~nm}$ pump pulse that creates on average $\langle N\rangle=0.1$ excitons per QD yields a transient absorption (TA) spectrum that shows the reduction of the bandedge absorption due to state filling at around 1,200 nm (see Fig. 1d). This band-edge bleach narrows down and loses intensity in the first nanoseconds after pumping. By comparing the difference between spectral cuts at $5 \mathrm{ps}$, when carrier cooling is complete, and $2.5 \mathrm{~ns}$, it follows that especially the bleach at the blue side of the first exciton transition is reduced (see Supplementary Section 3). In view of the tight-binding calculations, we attribute this change to the depletion of the higher-energy, higher-oscillator-strength $\mathrm{X}_{2}$ manifold in favour of the lower-energy, lower-oscillator-strength $\mathrm{X}_{1}$. The $\mathrm{X}_{2}-\mathrm{X}_{1}$ relaxation happens on a 700 ps timescale, a decay component also found in the time-resolved photoluminescence traces (see Supplementary Section 3). We thus conclude that the slow dynamics of the first exciton bleach reflect hole relaxation between the $\mathrm{VB}_{2}$ and $\mathrm{VB}_{1}$ states.

\section{Nearly thresholdless gain}

A most striking observation is the long-wavelength extension of the transient absorption bleach, covering the wavelength range of the sub-bandgap emission. This is better appreciated by looking 

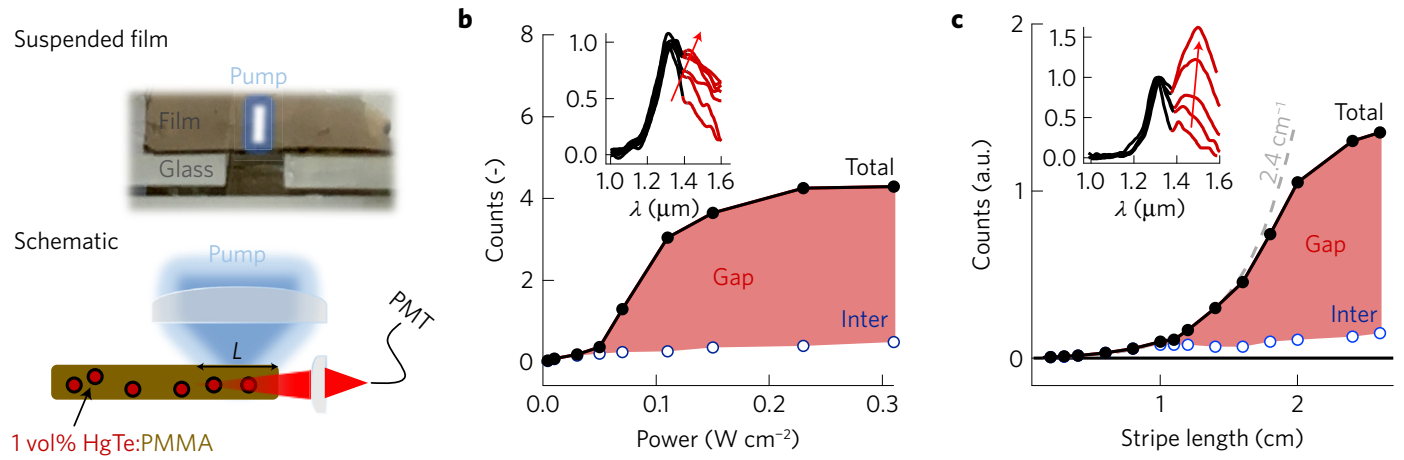

Figure 3 | Optical gain in hybrid PMMA-1\%HgTe nanocomposites under continuous-wave (CW) excitation at $447 \mathrm{~nm}$. a-c, Free-standing thin films are used for studying amplified spontaneous emission with a stripe excitation profile (a), both for increasing pump power (b) and stripe length $L$ (c). Supralinear increase of the light emitted at the long-wavelength side of the spectrum (indicated in red on normalized spectra in insets) is clearly observed, both for increasing pump power and stripe length. We note that both the total light intensity and the decomposed gap contribution show this behaviour. A threshold of $40 \mathrm{~mW} \mathrm{~cm}^{-2}$ is determined and, from the stripe-length-dependent measurements, a net modal gain coefficient of $2.4 \mathrm{~cm}^{-1}$ is extracted (for an excitation level of $100 \mathrm{~mW} \mathrm{~cm}^{-2}$ ).

the gain obtained in commonly used erbium-doped fibre amplifiers (EDFA) $\left(10^{-2}-10^{-3} \mathrm{~cm}^{-1}\right)$ and that of epitaxially grown III-V semiconductors $\left(\sim 10^{3} \mathrm{~cm}^{-1}\right)^{37,38}$ and colloidal QDs $\left(\sim 10^{3} \mathrm{~cm}^{-1}\right)^{39}$.

\section{Amplified spontaneous emission in nanocomposites}

The gain coefficients and the lifetime of the population inversion should suffice for stimulated emission to amplify spontaneous emission (ASE) in thin films of $\mathrm{HgTe} \mathrm{QDs}^{21}$. To verify this idea, we prepared nanocomposite films of HgTe QDs dispersed in polymethyl methacrylate (PMMA) at QD volume fractions of $0.1 \%$ and $1 \%$ (see Fig. 3a and Methods). In full agreement with HgTe QDs dispersed in $\mathrm{C}_{2} \mathrm{Cl}_{4}$, TA maps of PMMA/HgTe films attest net amplification at the long-wavelength side of the band-edge transition and their PL spectrum features two emission bands, with the high-energy band corresponding to the band-edge emission (see Supplementary Section 7). As further shown in Supplementary Section 7, pumping $1 \% \mathrm{PMMA} / \mathrm{HgTe}$ films with $110 \mathrm{fs}$ amplified pulses at $400 \mathrm{~nm}$ results in a marked enhancement of the long-wavelength emission band with increasing fluence. In addition, a clear supralinear increase of the total light intensity beyond $6 \mu \mathrm{Jcm}^{-2}$ is observed, which can be evaluated even further by decomposing the emission spectrum into an interband and a 'gap-state' component. Most remarkably, a similar behaviour is observed under CW pumping using a $447 \mathrm{~nm}$ laser diode (see Fig. 3b and Methods). Under such conditions, amplification of the gap-state emission is observed when pump powers exceed approximately $40 \mathrm{~mW} \mathrm{~cm}^{-2}$. In stark contrast with the behaviour of $1 \%$ PMMA/HgTe nanocomposites, the emission spectrum of $0.1 \%$ nanocomposites is independent of the pump power (see Supplementary Section 7). Hence, the amplification of the gap-state emission in $1 \%$ nanocomposites is not a spurious photo-induced effect caused by, for example, QD charging.

We analysed the amplification of the gap-state emission more quantitatively by variable stripe length (VSL) measurements using a set-up outlined in Fig. 3a. Using femtosecond pumping at a fixed fluence of $10 \mu \mathrm{J} \mathrm{cm}^{-2}$, we observe a supralinear increase of the total light output with increasing excitation stripe length (see Supplementary Section 7). The variation of the spectra as a function of stripe length already indicates that this reflects the amplification of the gap-state emission, a conclusion that is confirmed by plotting the gap-state emission intensity as a function of stripe length. Again, these characteristics are even more prominent in VSL measurements under CW pumping. As shown in Fig. 3c, the total light output shows a pronounced supralinear increase that concurs with an intensifying and narrowing gap-state emission-two clear signatures of ASE. From the exponential increase of the gap-state emission intensity, we obtain a CW gain coefficient of $2.4 \mathrm{~cm}^{-1}$ under the given pump conditions. As shown in Supplementary Section 8, this result is consistent with the CW pump power used and the optical characteristics derived from photoluminescence and transient absorption spectroscopy. This observation constitutes the first demonstration of CW pumped ASE by colloidal zero-dimensional QDs and it shows that CW gain by colloidal semiconductors is possible at extremely low pump thresholds, thanks to a combination of low-threshold excitation density and a long populationinversion lifetime. The threshold fluence of $40 \mathrm{~mW} \mathrm{~cm}^{-2}$ found here can be directly compared to the approximately $5,000 \mathrm{~mW} \mathrm{~cm}^{-2}$ threshold reported for CW ASE in films of two-dimensional CdSe nanoplatelets-the lowest threshold reported to date $e^{25,29}$.

\section{HgTe QDs as 4-level systems}

HgTe QDs exhibit optical gain well below the one-exciton-per-QD limit, as evidenced by their low fluence threshold for gain and ASE and the long-lived population inversion. Moreover, especially at the long-wavelength edge of the gain window, the emitting transition involved has no counterpart in absorption at that specific wavelength. While such a condition can be achieved with socalled 4-level systems, it cannot be simply met by the QD bandgap transition. This results in the need for multi-exciton gain or, at best, thresholds above $\langle N\rangle=2 / 3$ for core/shell QDs engineered to show single-exciton gain ${ }^{22}$. A further lowering of the gain threshold can result when stimulated emission is downshifted from the band-edge absorption by, for example, electron-phonon coupling ${ }^{40}$. Alternatively, almost thresholdless gain may result from emitting transitions of a band-edge carrier to a localized state in the QD bandgap that lack a counterpart in absorption. Although it is well known that localized bandgap states can be deliberately introduced in QDs by doping ${ }^{41}$, or may result from incomplete surface passivation ${ }^{42}$, optical gain via such states has never been reported.

As shown in Fig. 1b,c, HgTe QDs feature a dual-band emission where the longer wavelength, sub-bandgap emission overlaps with the observed optical gain window. This implies that the subbandgap stimulated emission in HgTe QDs cannot result from the band-edge emission being red-shifted by electron-phonon coupling, as this should lead to a single, red-shifted emission band. Sub-bandgap photoluminescence may, however, involve transitions of CB electrons to empty gap states or of VB holes to occupied gap states. The former is more likely in the present case (see below). As the same transition can give rise to stimulated emission, optical gain in HgTe QDs could be interpreted through a singleelectron level scheme as shown in Fig. 4a, where optical gain is related to a transition of a $\mathrm{CB}$ electron to a shallow, empty state in the bandgap. 


\section{Stimulated emission through surface traps}

To further identify the origin of the gap state, we analysed the optical gain of a given batch of $\mathrm{HgTe}$ QDs after successive purification cycles (see Methods and Supplementary Section 9). As shown in Fig. 4c, increased washing leads to a drop in photoluminescence quantum yield and a concomitant supralinear increase of the gain cross-section of an ensemble of HgTe QDs. Nuclear magnetic resonance (NMR) spectroscopy and elemental analysis indicates that purification results in a joint loss of both $\mathrm{Hg}$ and surface ligands from the QDs, possibly as mercury thiolate complexes.

The removal of such Z-type ligands ${ }^{43}$ is well known to introduce surface trap states in $\mathrm{CdX}(\mathrm{X}=\mathrm{S}, \mathrm{Se}, \mathrm{Te}) \mathrm{QDs}^{44-46}$. Following the approach of Houtepen et al. ${ }^{46}$ (see Methods and Supplementary Section 10), we used density functional theory (DFT) to construct a $\sim 2.0 \mathrm{~nm}$ model of a zinc-blende HgTe QD capped with methane thiolate. Figure $4 \mathrm{c}$ shows the energy level spectrum of this $\mathrm{HgTe}$ model QD, where the colours measure the projection of each state on different atom/fragment types. Most importantly, upon removal of one to four Z-type $\mathrm{Hg}\left(\mathrm{SCH}_{3}\right)_{2}$ ligands from the surface, a manifold of mostly localized gap states develops at $\sim 0.1 \mathrm{eV}$ above top of the valence band; in close agreement with the experimental observations. Similar to what was predicted for CdX, these states originate from the $5 p$ lone pair of two-coordinated tellurium surface atoms. As shown in Supplementary Section 10, DFT predicts that the oscillator strength of the HgTe gap-state transition exceeds that of the corresponding transitions in CdTe and CdSe QDs by two orders of magnitude, which can be linked to the stronger delocalization of the two-coordinated Te surface state in HgTe. Hence, while surface states linked to two-coordinated surface chalcogenides may be ubiquitous in II-VI QDs, the balance between the oscillator strength of the gap-state transition and spurious photo-induced absorption seems key to attain optical gain.

In summary, HgTe QDs exhibit ultralow threshold optical gain and amplification of spontaneous emission, which we attribute to a transition between the conduction-band edge and an empty surfacelocalized gap state. This makes HgTe QDs a 4-level system, for which population inversion can be reached at pump fluences substantially below the one-exciton-per-QD level. The gain and ASE thresholds observed here are up to two orders of magnitude lower than in recent literature reports $22,23,25$. Especially the demonstration of continuouswave ASE at a $40 \mathrm{~mW} \mathrm{~cm}^{-2}$ threshold stands out. Such thresholds match the power density of unconcentrated sunlight and correspond to an electrical DC density of approximately $20 \mathrm{~mA} \mathrm{~cm}^{-2}$, a level attained in state-of-the-art DC-driven, electroluminescent QD$\mathrm{LEDs}^{30,47,48}$. We thus conclude that the gain characteristics of $\mathrm{HgTe}$ QDs demonstrated here introduce an approach to QD-based gain materials that offers unique prospects of low $\mathrm{CW}$ lasing thresholds, spectral tuning and solution-processing.

\section{Methods}

Methods, including statements of data availability and any associated accession codes and references, are available in the online version of this paper.

\section{Received 24 March 2017; accepted 1 September 2017; published online 9 October 2017}

\section{References}

1. Yin, Y. \& Alivisatos, P. Colloidal nanocrystal synthesis and the organic-inorganic interface. Nature 437, 664-670 (2005).

2. Kovalenko, M. V. et al. Prospects of nanoscience with nanocrystals. ACS Nano 9, 1012-1057 (2015).

3. Keuleyan, S., Lhuillier, E., Brajuskovic, V. \& Guyot-Sionnest, P. Mid-infrared HgTe colloidal quantum dot photodetectors. Nat. Photon. 5, 489-493 (2011).

4. Bao, J. \& Bawendi, M. G. A colloidal quantum dot spectrometer. Nature 523, $67-70$ (2015)
. Wang, X. et al. Tandem colloidal quantum dot solar cells employing a graded recombination layer. Nat. Photon. 5, 480-484 (2011).

6. Kim, T. et al. Full-colour quantum dot displays fabricated by transfer printing. Nat. Photon. 5, 176-182 (2011).

7. Beard, M. C. et al. Comparing multiple exciton generation in quantum dots to impact ionization in bulk semiconductors: implications for enhancement of solar energy conversion. Nano Lett. 10, 3019-3027 (2010).

8. Galland, C. et al. Two types of luminescence blinking revealed by spectroelectrochemistry of single quantum dots. Nature 479, 203-207 (2011).

9. De Geyter, B. et al. The different nature of band edge absorption and emission in colloidal PbSe/CdSe core/shell quantum dots. ACS Nano 5, 58-66 (2011).

10. Norris, D. J., Efros, A. L. \& Erwin, S. C. Doped nanocrystals. Science 319, 1776-1779 (2008).

11. Aubert, T. et al. Homogeneously alloyed CdSeS quantum dots: an efficient synthesis for full optical tunability. Chem. Mater. 25, 2388-2390 (2013).

12. Nurmikko, A. What future for quantum dot-based light emitters? Nat. Nanotech. 10, 1001-1004 (2015).

13. Jenkins, A. Silicon lasers: the final frontier. Nat. Photon. 1, 240 (2007).

14. Simply silicon. Nat. Photon. 4, 491 (2010).

15. Chen, R. et al. Nanolasers grown on silicon. Nat. Photon. 5, 170-175 (2011).

16. Wang, Z. et al. Room temperature InP DFB laser array directly grown on (001) silicon. Nat. Photon. 9, 837-842 (2015).

17. Franzo, G., Priolo, F., Coffa, S., Polman, A. \& Carnera, A. Room-temperature electroluminescence from Er-doped crystalline Si. Appl. Phys. Lett. 64, 2235-2237 (1994).

18. Daldosso, N. et al. Erbium and silicon nanocrystals for light amplification. Conf. Proc.-Lasers and Electro-Optics Society Annual Meeting-LEOS 933-934 (2007).

19. Geiger, R. et al. Analysis of enhanced light emission from highly strained germanium microbridges. Nat. Photon. 7, 466-472 (2013).

20. Xie, W. et al. On-chip integrated quantum-dot silicon-nitride microdisk lasers. Adv. Mater. 29, 1604866 (2017).

21. Klimov, V. I. et al. Optical gain and stimulated emission in nanocrystal quantum dots. Science 290, 314-317 (2000).

22. Klimov, V. I. et al. Single-exciton optical gain in semiconductor nanocrystals. Nature 447, 441-446 (2007).

23. Dang, C. et al. Red, green and blue lasing enabled by single-exciton gain in colloidal quantum dot films. Nat. Nanotech. 7, 335-339 (2012).

24. Schaller, R. D., Petruska, M. a. \& Klimov, V. I. Tunable near-infrared optical gain and amplified spontaneous emission using PbSe nanocrystals. J. Phys. Chem. B 107, 13765-13768 (2003).

25. Grim, J. Q.et al. Continuous-wave biexciton lasing at room temperature using solution-processed quantum wells. Nat. Nanotech. 9, 891-895 (2014).

26. Klimov, V. I. Quantization of multiparticle auger rates in semiconductor quantum dots. Science 287, 1011-1013 (2000).

27. Chen, Y. et al. Flexible distributed-feedback colloidal quantum dot laser. Appl. Phys. Lett. 99, 241103 (2011).

28. García-Santamaría, F. et al. Suppressed auger recombination in 'giant' nanocrystals boosts optical gain performance. Nano Lett. 9, 3482-3488 (2009).

29. Fan, F. et al. Continuous-wave lasing in colloical quantum dot solids enabled by facet-selective epitaxy. Nature 544, 75-79 (2017).

30. Caruge, J. M., Halpert, J. E., Wood, V., Bulović, V. \& Bawendi, M. G. Colloidal quantum-dot light-emitting diodes with metal-oxide charge transport layers. Nat. Photon. 2, 247-250 (2008).

31. Keuleyan, S., Kohler, J. \& Guyot-Sionnest, P. Photoluminescence of mid-infrared HgTe colloidal quantum dots. J. Phys. Chem. C 118, 2749-2753 (2014).

32. Keuleyan, S., Lhuillier, E. \& Guyot-Sionnest, P. Synthesis of colloidal HgTe quantum dots for narrow mid-IR emission and detection. J. Am. Chem. Soc. 133, 16422-16424 (2011).

33. Kim, S. et al. Bandgap engineered monodisperse and stable mercury telluride quantum dots and their application for near-infrared photodetection. J. Mater. Chem. 21, 15232-15236 (2011).

34. Allan, G. \& Delerue, C. Tight-binding calculations of the optical properties of HgTe nanocrystals. Phys. Rev. B 86, 165437 (2012).

35. Keuleyan, S. E., Guyot-sionnest, P., Delerue, C. \& Allan, G. Mercury telluride colloidal quantum dots: electronic structure, size-dependent spectra, and photocurrent detection up to $12 \mu \mathrm{m}$. ACS Nano 8, 8676-8682 (2014).

36. Hens, Z. \& Moreels, I. Light absorption by colloidal semiconductor quantum dots. J. Mater. Chem. 22, 10406-10415 (2012).

37. Kittel, C. Introduction to Solid State Physics (John Wiley, 2005).

38. Jiang, S. B. et al. $\mathrm{Er}^{3+}$-doped phosphate glasses for fiber amplifiers with high gain per unit length. J. Non-Cryst. Solids 263, 364-368 (2000).

39. Pietryga, M. et al. Spectroscopic and device aspects of nanocrystal quantum dots. Chem. Rev. 116, 10513-10622 (2016).

40. Geiregat, P., Allan, G., Hens, Z. \& Delerue, C. Single-exciton optical gain in semiconductor nanocrystals: positive role of electron-phonon coupling. Phys. Rev. B 93, 115416 (2016). 
41. Viswanatha, R., Brovelli, S., Pandey, A., Crooker, S. \& aKlimov, V. I. Copper-doped inverted core/shell nanocrystals with 'permanent' optically active holes. Nano Lett. 11, 4753-4758 (2011).

42. Boehme, S. C. et al. Density of trap states and Auger-mediated electron trapping in CdTe quantum-dot solids. Nano Lett. 15, 3056-3066 (2015).

43. De Roo, J., Van Driessche, I., Martins, J. C. \& Hens, Z. Colloidal metal oxide nanocrystal catalysis by sustained chemically driven ligand displacement. Nat. Mater. 15, 517-521 (2016).

44. Hassinen, A. et al. Short-chain alcohols strip X-type ligands and quench the luminescence of $\mathrm{PbSe}$ and $\mathrm{CdSe}$ quantum dots, acetonitrile does not. J. Am. Chem. Soc. 134, 20705-20712 (2012).

45. Anderson, N. C., Hendricks, M. P., Choi, J. J. \& Owen, J. S. Ligand exchange and the stoichiometry of metal chalcogenide nanocrystals: spectroscopic observation of facile metal-carboxylate displacement and binding. J. Am Chem. Soc. 135, 18536-18548 (2013).

46. Houtepen, A., Hens, Z., Owen, J. S. \& Infante, I. On the origin of surface traps in colloidal II-VI semiconductor nanocrystals. Chem. Mater. 29, 752-761 (2017).

47. Kim, T.-H. et al. Full-colour quantum dot displays fabricated by transfer printing. Nat. Photon. 5, 176-182 (2011).

48. Kwak, J. et al. Bright and efficient full-color colloidal quantum dot light-emitting diodes using an inverted device structure. Nano Lett. 12, 2362-2366 (2012).

\section{Acknowledgements}

This research is funded by Ghent University (Special Research Fund BOF), BelSPo (IAP 7.35, photonics@be), EU-FP7 (Navolchi), NWO (Vidi grant, No. 723.013.002) Horizon
2020 ITN Phonsi and ERC-ULPICC and ERC-PoC Interdot. P.G. acknowledges the FWO Vlaanderen for a postdoctoral fellowship. S. Flamee is acknowledged for TEM imaging of the QDs and R. Van Deun and P. Smet are acknowledged for the use of the steady-state and time-resolved photoluminescence set-up and the cryogenic spectroscopy, respectively.

\section{Author contributions}

P.G. carried out the steady-state and time-resolved photoluminescence, and the ultrafast experiments, analysed the data, aided in theory development and wrote the manuscript; A.J.H. supervised the experiments, aided in theory discussions and wrote the manuscript: I.I. and F.Z. performed the DFT calculations and wrote the manuscript; L.K.S. synthesized the HgTe QDs and performed structural characterization (TEM, XRD); C.D. and G.A. carried out the tight-binding simulations and aided in theory development; D.V.T. aided in theory discussions and supervised the research; Z.H. initiated and supervised the research, aided in the theory development and wrote the manuscript.

\section{Additional information}

Supplementary information is available in the online version of the paper. Reprints and permissions information is available online at www.nature.com/reprints. Publisher's note: Springer Nature remains neutral with regard to jurisdictional claims in published maps and institutional affiliations. Correspondence and requests for materials should be addressed to P.G.

\section{Competing financial interests}

The authors declare no competing financial interests. 


\section{Methods}

Synthesis of HgTe quantum dots and composites. Colloidal HgTe QDs were synthesized by modifying the procedure of Keuleyan et al. ${ }^{32}$ and Sungwoo and colleagues ${ }^{33}$. As such, $270 \mathrm{mg}(1 \mathrm{mmol})$ of $\mathrm{HgCl}_{2}, 1.6 \mathrm{ml}(6 \mathrm{mmol})$ of dodecanethiol (DDT) and $8 \mathrm{ml}$ of oleylamine (OLA) were mixed in a $25 \mathrm{ml}$ flask and degassed under vacuum at $100^{\circ} \mathrm{C}$ for one hour, after which the reaction mixture was placed under nitrogen while keeping the temperature at $60^{\circ} \mathrm{C}$. Next, $1 \mathrm{ml}$ of a $1 \mathrm{M}$ solution of tellurium in tri-octylphosphine was rapidly injected and a black-coloured reaction mixture was obtained within a few seconds. The reaction mixture was kept at the injection temperature to allow for QD growth for $1 \mathrm{~min}(1,220 \mathrm{~nm}$ emission) to $3 \mathrm{~min}$ (1,300 $\mathrm{nm}$ emission), after which the reaction was quenched by injecting $10 \mathrm{ml}$ of toluene and cooling with a water bath. As-obtained HgTe QDs were purified twice using toluene and methanol. PMMA or polymethyl methacrylate, was purchased from Sigma Aldrich with a molecular weight of 120,000 and a mass density of $1.19 \mathrm{~g} \mathrm{~cm}^{-3} .0 .96 \mathrm{~g}$ of PMMA was dissolved in $10 \mathrm{ml}$ of toluene to obtain a $5 \mathrm{~m} \%$ solution. From this stock solution, $1 \mathrm{ml}$ was mixed with a given $\mathrm{HgTe}$ QD solution in toluene to obtain a volume fraction of QDs of 0.1 to $1 \%$ relative to PMMA. These composites were dropcast or spincoated on glass substrates to achieve different film thicknesses of a few hundreds of nanometres to several micrometres. We use PMMA composites since dropcasting or spincoating HgTe-only dispersions does not provide thick and optically smooth films. The composite approach also allows one to tune the volume fraction, which is useful to obtain a reference sample for the ASE experiments.

Linear optical properties. Lifetime measurements were carried out using a Hamamatsu near-infrared photomultipler tube in combination with a pulsed $\mathrm{Nd}$ :YAG laser operated at $532 \mathrm{~nm}$. Excitation pulses were $9 \mathrm{~ns}$ at a repetition rate of $10 \mathrm{~Hz}$. Samples were prepared by diluting a solution of QDs in dry tetrachloroethylene (Aldrich) to an optical density of 0.1 at the excitation wavelength of $650 \mathrm{~nm}$. The average lifetime is defined as $\langle t\rangle=\left(\int t I(t) \mathrm{d} t\right) /\left(\int I(t)\right)$. The quantum yield is determined using an integrating sphere method which requires no standard dye, thus resolving a common issue for near-infrared emitters.

Tight-binding calculations. The electronic structure and the optical properties of the HgTe QDs have been studied theoretically following closely the methodology described by Delerue and colleagues ${ }^{34}$. Details can be found in Supplementary Section 2.

DFT modelling. A non-stoichiometric $[\mathrm{HgTe}]_{55}\left(\mathrm{Hg}\left(\mathrm{SCH}_{3}\right)_{2}\right)_{13}$ QD of about $2.0 \mathrm{~nm}$ in diameter was prepared from the bulk zinc-blende crystal. The electronic structure and density of states of the pristine and Z-type displaced QDs have been computed at the DFT level of theory using the Perdew, Burke, and Ernzerhof (PBE) exchange-correlation functional ${ }^{49}$ using the CP2k 3.0 code $^{50}$. All structures were fully relaxed in vacuum. Oscillator strengths were calculated from the matrix elements of the position operator. More in-depth details on the DFT simulations are in Supplementary Section 10.
Ultrafast pump-probe spectroscopy. Samples were pumped using 180 femtosecond pulses at $700 \mathrm{~nm}$, created from the $1,028 \mathrm{~nm}$ fundamental (Pharos SP, $6 \mathrm{~W}$, Light Conversion) through nonlinear frequency mixing in an OPA (Orpheus, Light Conversion). Broadband probe pulses were generated in a sapphire crystal using the $1,028 \mathrm{~nm}$ fundamental. The pulses were delayed relative to the probe using a delay stage with maximum delay of $2.5 \mathrm{~ns}$ (Helios Spectrometer, Ultrafast Systems). Noise levels of $0.02 \mathrm{mOD}$ (RMS) are achieved by integrating the measurements over $1 \mathrm{~s}$ (or 2,500 shots). The probe spectrum covers the visible-near-infrared window from $450 \mathrm{~nm}$ up to 1,620 nm. HgTe QDs were dispersed in a transparent solvent (tetrachloroethylene) to achieve optical densities of 0.1 at the first exciton transition typically. Samples were stirred during all measurements. No air-free sample handling was required as $\mathrm{HgTe}$ is, under our conditions, insensitive to oxidation, as is evidenced by a lack of PL peak shift or change in photoluminescent quantum yield when exposing samples to ambient conditions for several weeks. The average number of absorbed photons (or photo-generated excitons) at time zero $\langle N\rangle$ was calculated as:

$$
\langle N\rangle=J_{\mathrm{ph}} \sigma_{700} \frac{1-10^{-A_{0,700}}}{A_{0,700}} \log (e)
$$

Here $J_{\mathrm{ph}}$ is the photon flux in photons $\mathrm{cm}^{-2}$ at $700 \mathrm{~nm}, \sigma_{700}$ is the absorption cross-section of the QDs at the pump wavelength of $700 \mathrm{~nm}$ and $A_{0,700}$ is the sample absorbance at $700 \mathrm{~nm}$. The beam size used for calculating $J_{\mathrm{ph}}$ is measured using a Thorlabs CCD Camera Beam profiler. The additional factor corrects for variation of the pump fluence along the pump beam path length. The cross-section $\sigma_{700}$ is determined starting from $\mu_{i, 400}$ (see Supplementary Information) $\mathrm{as}^{38}$ :

$$
\sigma_{700}=V_{\mathrm{QD}} \mu_{i, 400} \frac{A_{0,700}}{A_{0,400}}
$$

Here, $V_{\mathrm{QD}}$ is the volume of the quantum dot. Note that the (relative) error on $V_{\mathrm{QD}}$ is $32 \%$ due to the size distribution obtained through TEM analysis. This allows us to determine the scaling factor between pump fluence $(\mathrm{mW})$ and absorbed photons for the TA experiments as $0.31 \pm 0.1 \mathrm{~mW}^{-1}$. This translates into an error of $32 \%$ on all mentioned $\langle N\rangle$ values. We note that the calculation of $\langle N\rangle$ in thin films is very similar to the solution case, yet as the OD of the film at the pump wavelength is high (about 0.5 ) the correction factor becomes important.

Data availability. The authors declare that the main data supporting the findings of this study are available within the article and its Supplementary Information files. Extra data are available from the corresponding author upon request.

\section{References}

49. Perdew, J. P., Burke, K. \& Ernzerhof, M. Generalized gradient approximation made simple. Phys. Rev. Lett. 77, 3865-3868 (1996).

50. Iannuzzi, M., Schiffmann, F. \& Vandevondele, J. CP2k: atomistic simulations of condensed matter systems. Wiley Interdiscip. Rev. Comput. Mol. Sci. 4, 15-25 (2014) 